\title{
Performance Analysis of Rectenna Using Closed Form Equation
}

\author{
M.N.V Pavan Kumar, K. Avinash Kumar, Dr. CH. Srinivasu \\ Asst. Prof, Dept of ECE, Dadi institute of Engg. and Tech., Anakaplle \\ Asst. Prof, DIET, ANKAPALLE \\ Principal, DIET, Anakapalle
}

\begin{abstract}
Electromagnetics has an important role in power and energy industry. In this paper, the concept of rectenna is introduced firstly. The times past of rectenna for wireless energy harvesting and transmission is then reviewed. Finally examples are employed to illustrate some rectenna design and measurement issues such as rectenna impedance matching and its conversion efficiency. It isalso shown that rectennas can harvest wireless energy efficiently under certain conditions and have the potential to become a power supplier for some special applications. A high-efficiency rectenna component has been calculated and experienced at $6.2 \mathrm{GHz}$ planned for applications involving microwavepower show. The dipole mast and filtering circuitry are printed on a thin duroid substrate. A silicon Schottky barrier mixer diode with a low fail voltage is used as the rectifying device. The rectenna component is tested inside a waveguide simulator and achieves an RF-to-dc conversion efficiency of $85 \%$ at an input power level of 55- $\mathrm{mW}$ and 347-load. Closed-form equations be agreed meant for the diode efficiency and effort impedance as a sense of payment RF power. Measured in addition to intended efficiency results are in first-class agreement. The mast and circuit design are base on a full-wave electromagnetic simulator. Next harmonic power levels are $19 \mathrm{~dB}$ down from the primary input power.
\end{abstract}

Keywords: Microwave Power, rectanna, RF Power, Electromagnetics

\section{Introduction}

In the quest of green energy, scientists have been in the pursuit of converting deep space solar energy into high power microwave energy. This microwave energy is transmitted to the earth by a high gain parabolic reflector and received and converted to DC power with rectenna [1]. Scientists believe that within the next few decades this method will solve world's energy crisis significantly and becomean alternative energy source for developing countries those cannot effort conventional energy sources. Rectenna is defined as rectifying antenna. A rectenna is capable of receiving microwave energy from space and converting the received microwave power back to usable low frequency or DC power. A basic rectenna consists of an antenna, a diode rectifier and a dc bypass filter. This work presents a rectenna designed at $2.4 \mathrm{GHz}$ unlicensed band. The development starts with the design of microstrip patch antenna, followed by the design ofa diode rectifier and a lowpass filter (LPF). The final output of the rectenna is de power.

The high transmitted microwave power is captured by alargearrayantenna. The antenna element of the array is an aperture joined microstrip patch antenna (AJMPA). The AJMPA is the most suitable candidate due to its low profile, light weight, better power handling capability in an array, each of integration with active devices, isolation between feedlines and antenna radiators.



Fig 1 Schematic of rectenna

Figure 1shows the total diagram illustration of a generic rectanna. Once the microwave authority is received by the individual transmitter, it passes on or after end on the road to end the important frequency rectify diodes. A HSMS 8202 Schottky diode is used to convert lofty frequency power to DC electrical energy. The diode has a adaptation gain of 0.44. After the victorious mean of ACMPA, the antenna is included with the 
rectifier diode followed by a lumped building block LPF. The final amount produced is a DC voltage collected by a DC Voltmeter.

Components for microwave-power transmission have traditionally been focused at $2.45 \mathrm{GHz}$. To reduce the transmitting and rectenna aperture areas and increase the transmission range, researchers at ARCO Power Technologies, Inc., Washington, DC, developed a 82\% efficient rectenna element at $35 \mathrm{GHz}$ by 1991 [9]. $36 \mathrm{GHz}$ was targeted due to a decrease in the atmospheric absorption around this frequency. However, components for generating high power at $35 \mathrm{GHz}$ are expensive and inefficient.

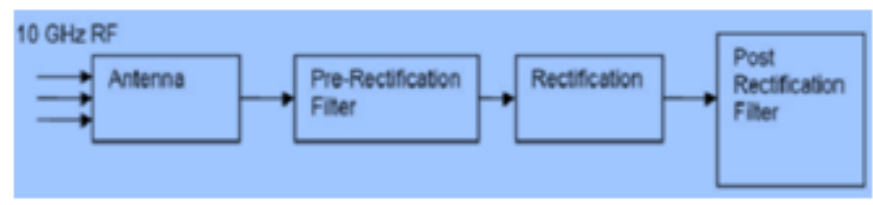

Fig 2 Block Diagram of rectenna

As the technology is growing the world is now moving toward wireless power. We can see that now days everyone prefers to use a wireless mouse or a wireless headphone. The make use of of batteries can make this possible excluding the predicament is that too many batteries are being used and there has to be a way by which these application canister lope wirelessly and the best thing would be if the batteries were not used. How can this be possible? This is the dilemma which we will try to solve in the aim The rectanna used will convert the RF power into de signal and as an alternative of batteries the claim will cover a rectanna en direction for produce the influence. consequently we will include a true wireless system, which has refusal ropes and no batteries. Although we have to agree that may be so power will not be produced by these rectanna but still as the expertise increase, the rectanna mechanized will also be improved. The word „rectenna' as we know today was first introduced by Brown. The basic concept of rectanna is a "rectifying antenna". In other word an antenna which will be used for receiving RF signal and a rectifying circuit which is used to give us DC power.

\section{Circular-Patch Antenna}

There are many candidate antenna designs, such as halfwave dipole, horn, parabolic antenna, and microstrip antenna. Some of these can be discarded for this application, due to their weight or size. A circular patch antenna was preferred, in part due to its capability of reducing the re radiation of harmonics. Figure 3 shows the response of a circular-patch antenna. Ideally, the return loss of a circularpatch antenna is about $50 \mathrm{~dB}$. In practice, the return loss is likely to be $12 \mathrm{~dB}$ to $22 \mathrm{~dB}$.

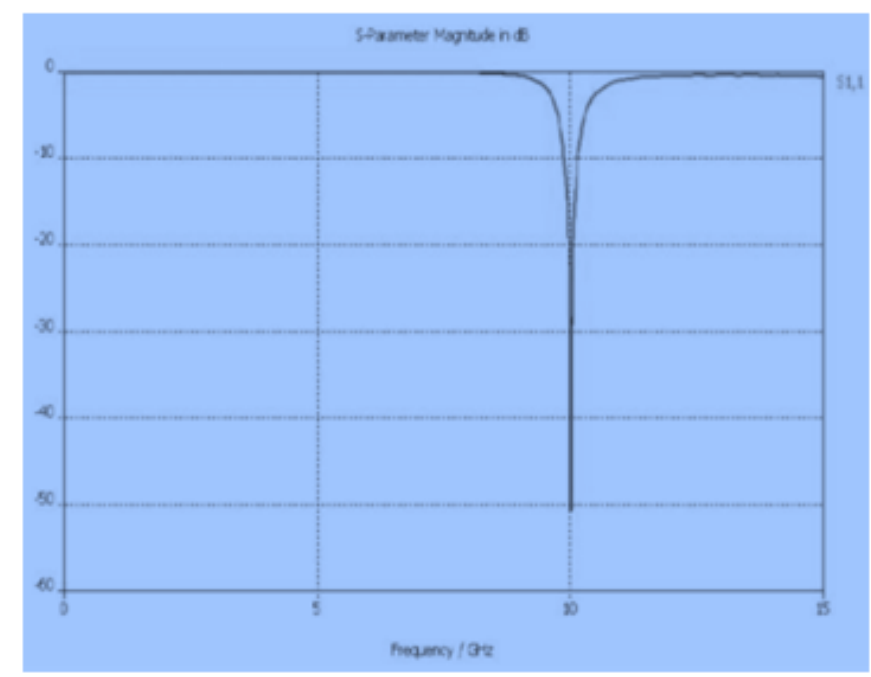

Fig 3 Response Curve of Circular-Patch Antenna

\section{Rectification Using Shotkey Diode}

Rectification is the key function of the rectenna: converting microwave rf energy into dc power. The Schottky diode was used as the rectifying device, due to a high switching capacity that enables it to follow a high-frequency input signal. A commercial Avago HSMS8101 was used in the NPS rectenna system. 
A post-rectification filter is used to extract the dc component and produce a smooth transient for output de power.



Fig 4 Response of the Schottky Diode

\section{Implementation Of Rectanna}

Rectifier is a nonlinear circuit, which converts RF power into DC power. The main characteristic of the operating effectiveness of a rectenna is its efficiency, determined by the losses, which arise during its conversion into DC power. The general block diagram of a conventional rectenna is shown in Fig. 2.The mathematical relation that describes the conversion efficiency is given by

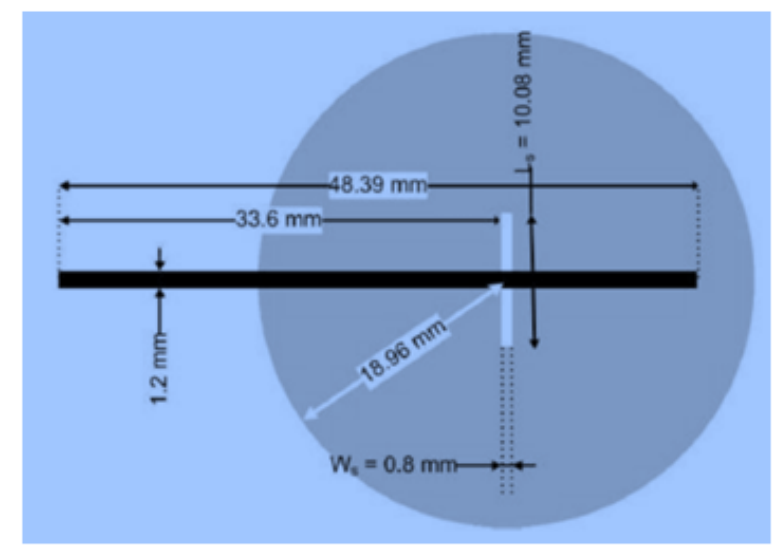

Fig 5 Circular Microstrip Patch Antenna Design

$$
\eta_{\text {rec } 1}=\frac{P_{D C}}{P_{r}}=\frac{V_{R_{C P}}^{2} / R_{C P}}{P_{r}}
$$

Antenna design is important in the proposed rectenna. The antenna absorbs the incident microwave power, and the rectifier converts it into a useful electric power. In this paper, in order to reduce the size of the rectenna, we eliminate the use of low pass filter.

\section{Outputs}

The full-wave rectenna without LPF meets the MAV requirement because of its higher conversion efficiency and constant output power. The simulated output dc power, voltage, and current of full-wave rectenna. The output power of the rectenna is $131 \mathrm{~mW}$ for an input power of $23 \mathrm{dBm}$, and the voltage oscillates from $2.58 \mathrm{~V}$ to $2.63 \mathrm{~V}$. The simulated conversion efficiency of the full-wave rectenna as a function of input power is shown in 
The full-wave design is able to convert microwave power to dc power with an efficiency of $63.9 \%$ at an input power of $190 \mathrm{~mW}$. The simulated output power of the full-wave rectenna design is shown in Figure6. The fullwave design is able to produce $112 \mathrm{~mW}$ at an input power of $200 \mathrm{~mW}$.

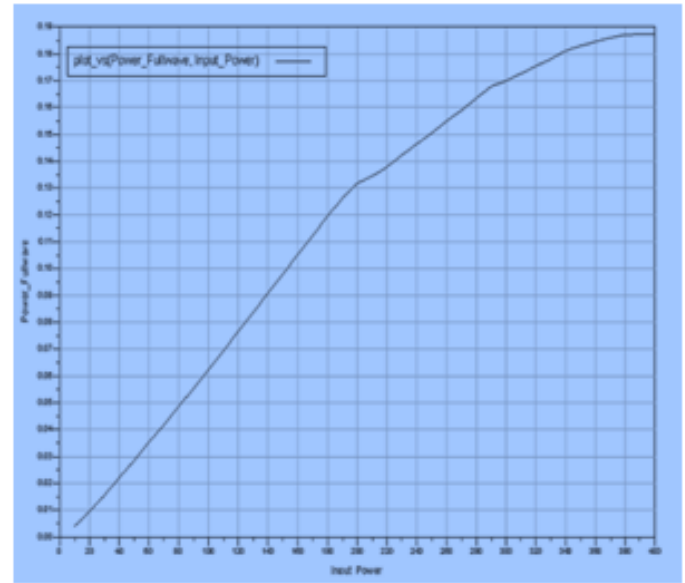

Fig 6 Conversion Efficiency of Final Full-Wave Rectenna Design.

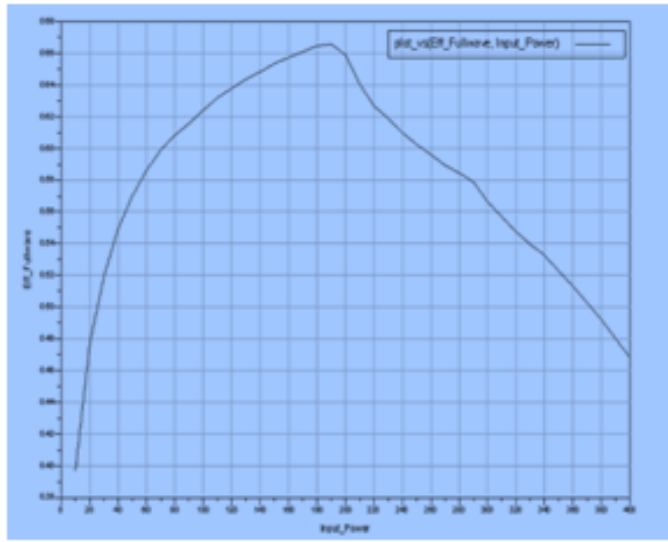

Fig 7 Output Power (watts) of Final Full-Wave Rectenna Design

\section{Conclusion}

In this paper, we have introduced the concept of rectennas and reviewed its history briefly. Some important design issues of rectennas have also been addressed. Moreover, a detailed discussion on the rectenna efficiency has been conducted. It is clear that to achieve high energy conversion efficiency, many parameters have to be taken into account. For low power density cases, high energy conversion efficiency may not be possible. Full wave rectification circuit designs were simulated using Agilent ADS software. A shorted stub microstrip tuner was introduced for impedance matching. The dipole mast and filtering circuitry are printed on a thin duroid substrate. A silicon Schottky barrier mixer diode with a low fail voltage is used as the rectifying device. The rectenna component is tested inside a waveguide simulator and achieves an RF-to-dc conversion efficiency of $85 \%$ at an input power level of $55-\mathrm{mW}$ and 347-load. Closed-form equations be agreed meant for the diode efficiency and effort impedance as a sense of payment RF power. Measured in addition to intended efficiency results are in first-class agreement. The mast and circuit design are base on a full-wave electromagnetic simulator. next harmonic power levels are $19 \mathrm{~dB}$ down from the primary input power.The circular-antenna array was selected to reduce the re-radiation pattern of the diode. A full-wave rectenna without low-pass filter was selected to reduce the weight of the rectenna element yet achieve high conversion efficiency. In addition, a harmonic balance analysis was conducted in order to compare the influence of the harmonics onvarious circuit designs. The full-wave rectenna circuit was simulated to verify performance, and the conversion efficiency. The performance of the WPT can be further enhanced by complementing it with a high-power transmitter and high-gain antenna to increase the power-density incident on the rectenna array. 


\section{Acknowledgement}

The authors of this paper would like to acknowledge Honble Correspondent Sri DADI Ratnakar garu for his immense support and Principal Dr. CH. Srinivasu for his suggestions. Above all, the extreme mental support and source of inspiration from HOD ECE Sri Vamsidhar Anagani Assoc.Prof and all the faculty members of ECE and friends are widely acknowledged.

\section{References}

[1]. Giuseppina Monti, Luciano Terricone and Michele Spartano, " X-band planar rectenna", IEEE Antenna and Wireless Propagation Letters, Vol.10,1116-1118,2011.

[2]. J.O. McSpadden, Lu Fan and Kai Chang, "Design and experiments of a high-conversion efficiency 5.8-GHz rectenna", IEEE Transactions on Microwave Theory and Techniques, VOL. 46. NO.12. 2053-2060, Dec. 1998.

[3]. L. Mateu, and F. Moll, "Review ofenergy harvesting techniques and applications for microelectronics", Proceedings of the SPIE Microtechnologies for the New Millennium, Sevilla, pp. 359-73. 2005.

[4]. R. J. Guttmann and R. B. Gworek."Yagi-uda receiving elements in microwave power transmission system rectennas". Journal of Microwave Power, 14(4):313-320, 1979.

[5]. L. Mateu, and F. Moll, "Review ofenergy harvesting techniques and applications for microelectronics", Proceedings of the SPIE Microtechnologies for the New Millennium, Sevilla, pp. 359-73. 2005.

[6]. Y. Aoki M. Otsuka T. Idogaki Shibata, T. and T. Hattori. "Microwave energy transmission system for microrobot." IEICE-Trans. Electr., 80-c(2):303-308, 1997.

[7]. Tae-Whan Yoo and Kai Chang, "Theoretical and experimental development of 10 and 35 GHz rectennas," IEEE Transactions on Microwave and Techniques,.40.NO.6.June.1992.

[8]. IEEE Microwave Magazine, Vol. 3, no. 4, December 2002. 